\title{
Effects and mechanism of the bile acid (farnesoid $X$ ) receptor on the Wnt/ $\beta$-catenin signaling pathway in colon cancer
}

\author{
JIAYU MAO ${ }^{1}$, XUEQI CHEN $^{1}$, CHUNSAIER WANG $^{1}$, WENBIN LI $^{1}$ and JINGNAN LI ${ }^{1,2}$ \\ ${ }^{1}$ Department of Gastroenterology, Peking Union Medical College Hospital, \\ Peking Union Medical College and Chinese Academy of Medical Science; \\ ${ }^{2}$ Key Laboratory of Gut Microbiota Translational Medicine Research, \\ Chinese Academy of Medical Science, Beijing 100730, P.R. China
}

Received June 19, 2019; Accepted February 28, 2020

DOI: $10.3892 / \mathrm{ol} .2020 .11545$

\begin{abstract}
The downregulation of farnesoid X receptor (FXR; gene name, nuclear receptor subfamily 1 group h member 4 ), an enteric nuclear bile acid receptor, has been reported in colorectal carcinoma (CRC), and FXR expression has been inversely correlated with CRC stage and clinical outcome. FXR knockdown in chronic colitis mouse models of intestinal tumorigenesis results in early mortality and increased tumor progression via promoting Wnt signaling. The aim of the present study was to explore the effects and mechanism of FXR on the Wnt/ $\beta$-catenin signal pathway in CRC. FXR and $\beta$-catenin protein expression levels were detected in an ulcerative colitis mouse model and human colon cancer cell lines (HT-29, Caco-2 and HCT-116). Gain- and loss-of-function studies were conducted by transfecting colon cancer cells with FXR siRNA and treating them with the FXR agonist GW4064. Subsequently, $\beta$-catenin transcriptional activity was measured using the dual-luciferase assay, and $\beta$-catenin/TCF4 complex levels and $\beta$-catenin protein and mRNA expression levels were determined. FXR and $\beta$-catenin expression levels were inversely associated in both the animal model and colon cancer cells. The Wnt signaling pathway was activated by increased $\beta$-catenin/TCF4 complex levels upon FXR silencing; however, mRNA and protein levels of $\beta$-catenin were not significantly affected. The FXR agonist GW4064 significantly inhibited the
\end{abstract}

Correspondence to: Dr Jingnan Li, Department of Gastroenterology, Peking Union Medical College Hospital, Peking Union Medical College and Chinese Academy of Medical Science, 1 Shuaifuyuan Building Group Dong Dan San Tiao Street, Beijing 100730, P.R. China

E-mail: 1ijn2008@126.com

Abbreviations: FXR, farnesoid X receptor; BA, bile acid; CRC, colorectal carcinoma; UC, ulcerative colitis; EMSA, electrophoretic mobility shift assay; NR, nuclear receptor; VDR, vitamin D receptor

Key words: colorectal neoplasms, FXR, Wnt/ $\beta$-catenin signaling pathway proliferation of cells but promoted the transcriptional activity of $\beta$-catenin. Thus, the present study demonstrated that FXR influences the Wnt/ $\beta$-catenin signaling pathway. Furthermore, loss of FXR expression promotes the transcriptional activity of $\beta$-catenin, whereas FXR activation results in the opposite effect.

\section{Introduction}

The incidence and mortality rates of colorectal cancer (CRC) vary markedly worldwide, due to heterogeneity in environmental and genetic factors. A high-fat and low-fiber diet has been established as a major risk factor for colon cancer development (1), and cholecystectomy has been associated with right-sided colon cancer (2). These factors may result in an increased bile acid (BA) load in the intestine and higher relative concentrations of fecal secondary Bas (3). Consequently, resistance to BA-induced DNA oxidative damage, inflammation, nuclear factor- $\kappa \mathrm{B}$ activation and enhanced cell proliferation has been reported to increase colon tumorigenesis (4).

BAs are endogenous ligands for the nuclear farnesoid X receptor (FXR; gene name, Nuclear Receptor Subfamily 1 Group H Member 4), which regulates BA concentrations by modulating BA influx, efflux and detoxification $(5,6)$. FXR is downregulated in both mouse and human models of CRC during the progression from normal intestinal epithelia to dysplastic lesions, and its expression has been inversely correlated with CRC stage and clinical outcome $(7,8)$. FXR silencing in chronic colitis mouse models of intestinal tumorigenesis results in early mortality and increased tumor progression (9).

The mechanism by which FXR suppresses tumor growth remains unclear, but it may involve protecting the colonic epithelium from inflammation and ameliorating BA toxicity by upregulating intracellular BA binding proteins and efflux transporters and downregulating influx transporters and de novo BA synthesis (10-12). However, FXR has additional antitumorigenic functions independent of BA homeostasis regulation (13). FXR deficiency increases colon cancer susceptibility by increasing epithelial permeability to bacteria, promoting $\mathrm{Wnt} / \beta$-catenin signaling and increasing intestinal inflammation (8). The aim of the present study was to explore the effects and mechanism of FXR on the Wnt/ $\beta$-catenin signaling pathway in CRC. 


\section{Materials and methods}

Cell culture. The human colon cancer cell lines HT-29, Caco-2 and HCT-116 were obtained from the State Key Laboratory of Molecular Oncology, National Cancer Center/Cancer Hospital, Chinese Academy of Medical Sciences (cell line not authenticated). HT-29, Caco-2 and HCT-116 cells were maintained in Dulbecco's modified Eagle's medium: Nutrient mixture F-12 media (DMEM/F-12; HyClone; GE Healthcare Life Sciences), minimum essential medium/Earle's balanced salt solution (MEM/EBSS; HyClone; GE Healthcare Life Sciences) and Iscove's modified Dulbecco's medium (IMDM; HyClone; GE Healthcare Life Sciences), respectively. They were supplemented with $10 \%$ fetal bovine serum (FBS; HyClone; GE Healthcare Life Sciences) and $1 \%$ penicillin/streptomycin (HyClone; GE Healthcare Life Sciences) at $37^{\circ} \mathrm{C}$ in a humidified incubator containing $5 \% \mathrm{CO}_{2}$. Caco-2 cells were co-treated with $1 \%$ non-essential amino acids (HyClone; GE Healthcare Life Sciences).

Plasmidandluciferase reporterassays. FXR siRNA (sc-38848) and non-targeting negative control siRNA (siNC) (sc-37007) were purchased from Santa Cruz Biotechnology, Inc. and transiently transfected into cells using Lipofectamine ${ }^{\circledR} 2000$ according to the manufacturer's protocol (Invitrogen; Thermo Fisher Scientific, Inc.). The sequences of these siRNAs was not available from the manufacturer. A preliminary experiment was conducted to and indicated $40 \mathrm{nM}$ as the optimal dose, and this was used for the subsequent experiments (data not shown). At $48 \mathrm{~h}$ after cells were transfected with $40 \mathrm{nM}$ siFXR or siNC, total protein was extracted and FXR was measured via western blotting. According to the physiological function of FXR in regulating BA metabolism, FXR activation primes the transcription of the small heterodimer partner (SHP), ultimately resulting in decreased expression of CYP7A1, the rate-limiting enzyme of BA de novo synthesis (14). Therefore, the target gene of FXR, SHP/NR0B2 was also measured to verify its successful knockdown.

E.coli $\mathrm{DH} 5 \alpha$, TOPflash and pRL-TK vectors were obtained from the State Key Laboratory of Molecular Oncology, National Cancer Center/Cancer Hospital, Chinese Academy of Medical Sciences. Luciferase assays were performed using the Dual-Luciferase Reporter assay system (Invitrogen; Thermo Fisher Scientific, Inc.). Briefly, $300 \mathrm{ng}$ of TOPflash and $30 \mathrm{ng}$ of pRL-TK with siFXR or siNC were co-transfected into cells using Lipofectamine ${ }^{\circledR} 2000$ (Invitrogen; Thermo Fisher Scientific, Inc.) according to the manufacturer's instructions. Luciferase activity in the harvested cell lysates was measured using a luminometer, $48 \mathrm{~h}$ after transfection. The results were normalized to Renilla luciferase activity.

Electrophoretic mobility shift assay (EMSA). Nuclear extracts from cultured cells were prepared according to the manufacturer's protocol with Nuclear and Cytoplasmic Extraction reagents (Pierce; Thermo Fisher Scientific, Inc.). The biotin-labelled Bio-TCF-4 probe (Bio-5'-CCCTTTGAT CTTACC-3') and cold-TCF-4 probe (cold-5'-AGTTGAGGG GACTTTCCCAGGC-3') $(15,16)$, were constructed by Sango Biotech Co., Ltd. EMSAs were performed by incubating $5 \mu \mathrm{g}$ nuclear extract with $0.5 \mu 1$ biotin-labelled Bio-TCF-4 DNA probes in a $15-\mu \mathrm{l}$ binding reaction mixture containing $1.5 \mu \mathrm{l} 10 \mathrm{X}$ binding buffer and $1 \mu \mathrm{l}$ poly $(\mathrm{dI}-\mathrm{dC}) /$ poly $(\mathrm{dI}-\mathrm{dC})\left(\right.$ LightShift $^{\mathrm{TM}}$ Poly(dI-dC), cat. no. 20148E, Pierce; Thermo Fisher Scientific, Inc.). Following incubation at room temperature for $20 \mathrm{~min}$, the samples were loaded onto a $6.5 \%$ polyacrylamide gel and electrophoresed in Tris/Borate/EDTA (TBE) buffer at $180 \mathrm{~V}$ for $60 \mathrm{~min}$ at $4^{\circ} \mathrm{C}$. For competition experiments, the nuclear extracts were preincubated with $2 \mu \mathrm{l}$ unlabeled oligonucleotides before the biotin-labeled oligonucleotide DNA probe was added. The complexes were transferred onto nitrocellulose in TBE buffer for $40 \mathrm{~min}$ at $390 \mathrm{~mA}$ at room temperature. Immunoreactive bands were developed using streptavidin-horseradish peroxidase (HRP) and visualized via autoradiography. CK represents the background control group with only the probe and no nuclear extract; 100x represents the competitive control group with additional non-labelled probe; + represents the positive control group [nuclear extracts from cells pre-treated with Wnt signaling activator BML284 (Abcam)] (17); C represents the control group pre-treated with siNC. Intensity analysis was performed to demonstrate the differences between groups, for which intensity of the positive control group was recorded as 1 and results of other groups were calculated as the intensity ratio relative to the positive control group. Analysis was performed using ImageJ (version 1.52q; National Institutes of Health).

Western blotting. Cultured cells were lysed in $500 \mu 1$ RIPA lysis buffer (Applygen Technologies, Inc.), and total protein concentration was determined using a BCA protein assay kit (Applygen Technologies, Inc.). Equal amounts of protein (50 $\mu \mathrm{g} / \mathrm{lane}$ ) were electrophoresed using 10\% SDS-PAGE gel and then transferred onto nitrocellulose membranes. After blocking with TTBS containing 5\% non-fat milk and $0.1 \%$ Tween 20 for $2 \mathrm{~h}$ at room temperature, the membranes were incubated overnight with a primary antibody at $4^{\circ} \mathrm{C}$. After washing with TTBS containing $0.1 \%$ Tween-20 3 times for 5 min each, the membranes were incubated with a HRP-labeled secondary antibody for another $2 \mathrm{~h}$ at room temperature. The membranes were then developed using Pierce ECL Western Blotting Substrate (Thermo Fisher Scientific, Inc.) and exposed using an Amersham Imager 600 (GE Healthcare). Primary anti-FXR mouse monoclonal antibody $(1: 100,000$, cat. no. ab187735, Abcam), anti- $\beta$-catenin rabbit monoclonal antibody (1:100,000, cat. no. ab32572, Abcam), anti- $\beta$-actin mouse monoclonal antibody (1:10,000, cat. no. sc-8432, Santa Cruz Biotechnology, Inc.), anti-Poly (ADP-ribose) polymerase (PARP) rabbit polyclonal antibody (1:1,000, cat. no. \#9542,) and secondary anti-mouse (1:10,000, cat. no. \#7076), anti-rabbit (1:10,000, cat. no. \#7074) horseradish peroxidase-conjugated antibodies (all Cell Signaling Technology, Inc.) were used for protein labeling. $\beta$-actin was used here as a loading control of total protein extract, and PARP was used as a loading control of nuclear protein extract (18).

RNA extraction and reverse transcription-quantitative (RT- $q)$ PCR analysis. RNA was isolated from cells using TRIzol ${ }^{\circledR}$ reagent according to the manufacturer's protocol (Invitrogen; Thermo Fisher Scientific, Inc.). cDNA was generated from $500 \mathrm{ng}$ total RNA using SuperScript II Reverse Transcriptase (Thermo Fisher Scientific, Inc.). RT-qPCR reactions were performed as 
follows: $25^{\circ} \mathrm{C}$ for $10 \mathrm{~min}, 50^{\circ} \mathrm{C}$ for $15 \mathrm{~min}$ and $85^{\circ} \mathrm{C}$ for $5 \mathrm{~min}$. qPCR analysis was performed using SYBR Green PCR master mix (Thermo Fisher Scientific, Inc.) and analyzed using a CFX96 real-time PCR system (Bio-Rad Laboratories, Inc.). The reaction conditions included an initial pre-denaturation step at $95^{\circ} \mathrm{C}$ for $30 \mathrm{sec}$, followed by 40 cycles of thermal steps consisting of $95^{\circ} \mathrm{C}$ for $5 \mathrm{sec}$ and $60^{\circ} \mathrm{C}$ for $30 \mathrm{sec}$. Values were normalized to GADPH. The fold-change was calculated by the $2^{-\Delta \Delta C q}$ method (19). The primer sequences were as follows: $\beta$-catenin (human) forward, 5'-AGA ACCCCTTGGATATCGCC-3' and reverse, 5'-TGG CCACCCATCTCATGTTC-3'; and GAPDH (human) forward, 5'-CTCTGCTCCTCCTGTTCGAC-3' and reverse, 5'-GCGCCC AATACGACCAAATC-3'.

Cell proliferation assay. The human colon cancer cell lines

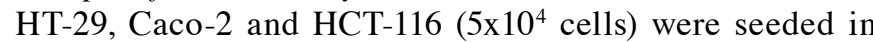
quintuplicate in 96 -well plates and incubated at $37^{\circ} \mathrm{C}, 95 \%$ humidity, and 5\% carbon dioxide overnight. At the indicated time points, $40 \mu 1$ MTT (Sangon Biotech Co., Ltd.) solution $(1 \mathrm{mg} / \mathrm{ml})$ was added to each well. The plates were incubated at $37^{\circ} \mathrm{C}$ for $1 \mathrm{~h}$. Subsequently, the medium was removed, and $200 \mu \mathrm{l}$ DMSO was added to each well to dissolve the formazan crystals. Next, the plates were measured at $490 \mathrm{~nm}$ on a multi-well plate reader. The effect of the FXR agonist GW4064 (Sigma-Aldrich; Merck KGaA) on cell proliferation was measured. Based on the present studies on GW4064 $(20,21)$, the experimental groups in the present study included: i) Different doses of GW4064 (1,3 and $5 \mu \mathrm{M})$; and ii) different durations of GW4064 treatment (12, 24, 36 and $48 \mathrm{~h}$ ). Control groups were treated with only 1,3 or $5 \mu \mathrm{M} \mathrm{GW} 4064$ according to the dose, and other conditions were consistent with the experimental groups.

Mice. Eight-week-old male C57BL/6 mice $(21 \pm 2 \mathrm{~g})$ were purchased from Vital River Laboratory Animal Technology Co. Ltd. All animals were housed under specific pathogen-free conditions in plastic cages (4-5 mice/cage) with free access to drinking water and a pellet-based diet, under controlled conditions of humidity (40-60\%), light (12/12 h, light/dark cycle) and temperature $\left(22 \pm 1^{\circ} \mathrm{C}\right)$ in the National Cancer Center/Cancer Hospital animal facilities. Experiments were performed in accordance with local laws and the Council of the European Communities Directive of 1986 (86/609/EEC) guidelines for the care and use of laboratory animals. All animal experiments were approved by and conducted in accordance with the recommendations of the Animal Care Ethics and Use Committee of Peking Union Medical College (Beijing, China; approval no. XHDW-2015-0032). All measures were made to minimize animal suffering and to reduce the number of animals used.

All mice $(n=30)$ were initially housed together ( 5 animals/cage) for adaption, one week before randomization into the control group [no ulcerative colitis (UC)-associated carcinogenesis induction, $\mathrm{n}=10$ ) and model group (UC-associated carcinogenesis, $\mathrm{n}=20$ ). For the UC carcinogenesis model, the mice were injected intraperitoneally with $12.5 \mathrm{mg} / \mathrm{kg}$ body weight azoxymethane (AOM). After one week, $2.5 \%$ dextran sulfate sodium (DSS) (MP Biomedicals, LLC) was added to the drinking water for 5 days, followed by 10 weeks and 2 days of regular drinking water. This modeling method was based on a novel inflammation-related mouse colon carcinogenesis model (22), indicating that 1-week administration of DSS after initiation with a low dose of AOM exerted a powerful tumor-promoting activity in colon carcinogenesis. Body weight, colitis symptoms and other animal behavior were recorded every week throughout the experiment. At the 12th week, all mice were sacrificed via injection of $200 \mathrm{mg} / \mathrm{kg}$ pentobarbital sodium to induce overdose anesthesia, and colon tissues were removed.

Histology and immunohistochemistry. Tissue specimens were fixed in $10 \%$ formalin at room temperature for $12-24 \mathrm{~h}$, dehydrated and paraffin embedded. Graded ethanol was used in a dehydration series as follows: $70 \%$ for $2 \mathrm{~h}, 80 \%$ for $2 \mathrm{~h}, 90 \%$ for $2 \mathrm{~h}, 95 \%$ for $2 \mathrm{~h}$, an two lots of $100 \%$ for $2 \mathrm{~h}$. The sections were deparaffinized in 5\% xylene and then dehydrated in ethanol as following: $100 \%$ for $1 \mathrm{~min}, 95 \%$ for $1 \mathrm{~min}$ and $75 \%$ for $1 \mathrm{~min}$. It was then washed with distilled water and stained with Harris hematoxylin at room temperature for $3 \mathrm{~min}$ and rinsed in distilled water. The section was differentiated with $1 \%$ acid alcohol, washed under running water, and counterstained with eosin at room temperature for $1 \mathrm{~min}$. At last, it was washed with water, dehydrated with ascending grades of ethanol, $95 \%$ for $1 \mathrm{~min}$, two lots of $100 \%$ for $1 \mathrm{~min}$, and cleaned with xylene. The mounted slides were then examined and images were captured with magnifications of $\mathrm{x} 4$, $\mathrm{x} 10$ and x20 using a light microscope (Olympus).

Standard immunohistochemical procedures were performed. Briefly, $5-\mu \mathrm{m}$-thick sections were treated with $3 \%$ hydrogen peroxide at room temperature for $5 \mathrm{~min}$ to quench endogenous peroxidase activity and subjected to antigen retrieval by boiling the slides in an antigen unmasking solution (Zsbio Commerce Store) for $15 \mathrm{~min}$, according to the manufacturer's instructions. The sections were incubated sequentially for $60 \mathrm{~min}$ at room temperature in 50\% non-immune serum (from the host animal in which the secondary antibody was raised) in PBS (to avoid non-specific signaling) and overnight at $4^{\circ} \mathrm{C}$ with the following primary antibodies: Anti-FXR rabbit polyclonal antibody (1:300, cat. no. sc-13063, Santa Cruz Biotechnology, Inc.) and anti- $\beta$-catenin rabbit monoclonal antibody (1:2,000, cat. no. ab32572, Abcam). The sections were washed for $10 \mathrm{~min}$ with PBS and incubated for $30 \mathrm{~min}$ at room temperature with the secondary biotinylated antibody (1:1, cat. no. PV-9000, Zsbio Commerce Store). After several washes with PBS ( 3 washes; 5 min each), the sections were incubated with the avidin-biotin complex (Cell Signaling Technology, Inc.) for $30 \mathrm{~min}$ at room temperature. After washing with PBS, the peroxidase reaction was developed by incubation with diaminobenzidine (Sigma-Aldrich; Merck KGaA) 8 min at room temperature. The staining was observed under a microscope, when the brown color appeared, all sections were immediately washed three times for $5 \mathrm{~min}$ with PBS to terminate the reaction simultaneously. Counterstaining was carried out with methylene-blue (Sigma-Aldrich; Merck $\mathrm{KGaA}$ ). For negative controls, $1 \%$ non-immune serum in PBS was used instead of the primary antibodies. The positively stained cells were counted in randomized fields at $\mathrm{x} 400$ magnification using a light microscope (Olympus).

FXR and $\beta$-catenin expression was qualitatively assessed through scored by two independent, experienced pathologists who were blinded to the clinicopathological 
A
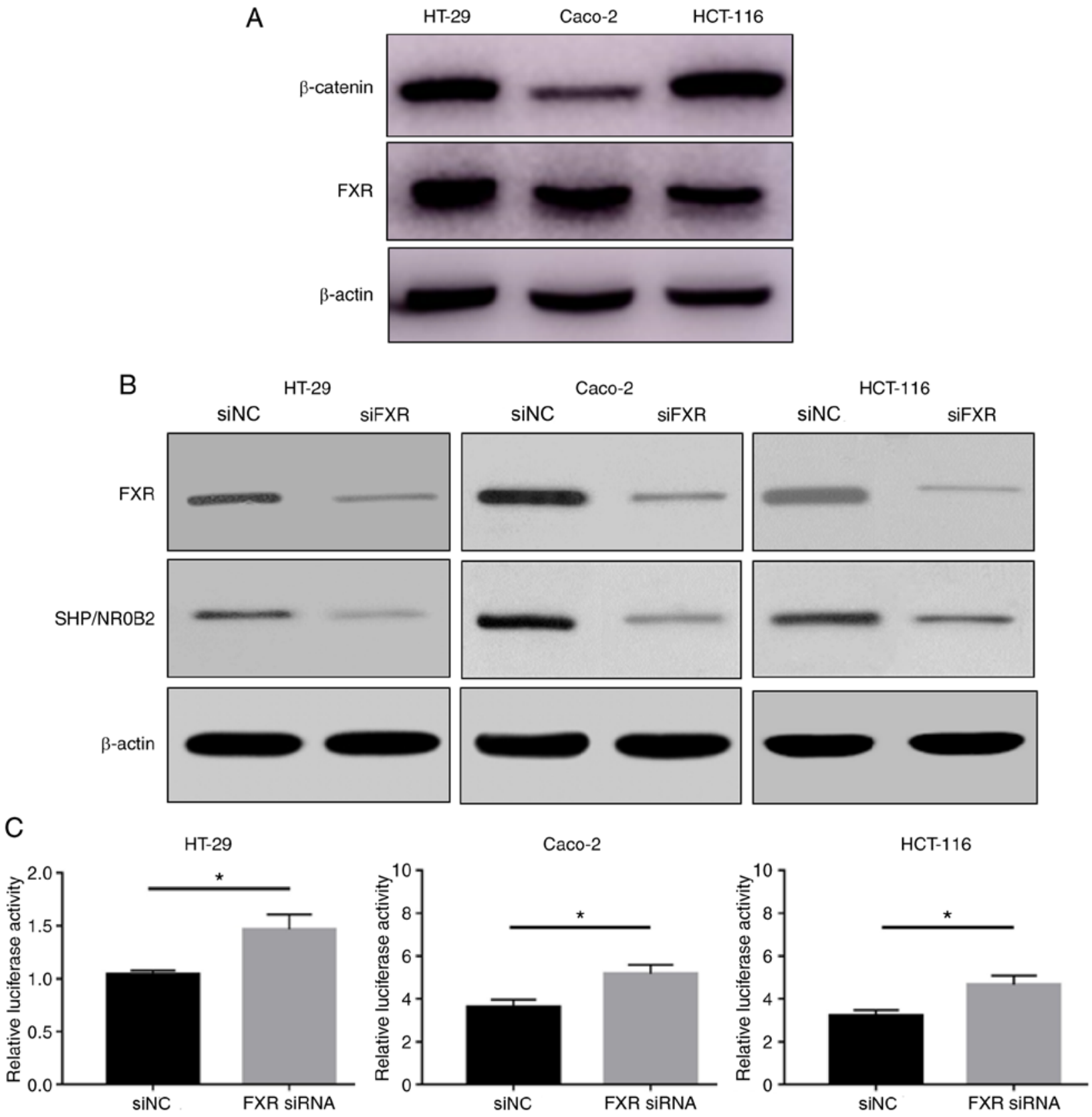

Figure 1. FXR knockdown activates the Wnt signaling pathway. (A) FXR and $\beta$-catenin protein expression levels detected by western blotting in the HT-29, Caco-2 and HCT-116 cell lines. $\beta$-actin was used as a loading control. (B) FXR silencing was confirmed in all cell lines. (C) Silencing FXR significantly increased $\beta$-catenin-mediated luciferase activity. The error bars represent the SEM of three independent samples. " $\mathrm{P}<0.05$ vs. siNC. FXR, farnesoid X receptor; si, small interfering; $\mathrm{NC}$, negative control.

information. Staining intensity was scored as (absent, 0), (weak, 1), (moderate, 2) and (strong, 3). The percentages of immunoreactive cells were grouped and scored manually as follows: $1,<25 \% ; 2,25-50 \% ; 3,50-75 \%$; and $4,>75 \%$. A final score was obtained by multiplication of the single scores using. There was $\sim 95 \%$ concordance between the two observers. Differences were resolved by consensus evaluation of the slides.

Statistical analysis. The results are expressed as the mean \pm SEM, as indicated in the figure legends. Statistical significance was determined by two-tailed independent Student's t-test or one-way ANOVA and Tukey's post hoc test. All statistical calculations were performed using the SPSS
22.0 software (IBM Corp.). $\mathrm{P}<0.05$ was considered to indicate a statistically significant difference.

\section{Results}

FXR knockdown activates the Wnt signaling pathway. FXR and $\beta$-catenin protein expression levels were determined in the HT-29, Caco-2 and HCT-116 cell lines. HT-29 and Caco-2 cells highly express FXR, and HT-29 and HCT-116 cells highly express $\beta$-catenin (Fig. 1A). FXR and $\beta$-catenin expression levels were different between these human colon cancer cell lines.

siFXR efficiently silenced FXR expression and its target gene SHP/NR0B2 (Fig. 1B). The effect of FXR on 
A

HT-29

Caco-2

HCT-116

CK C siFXR 100x +

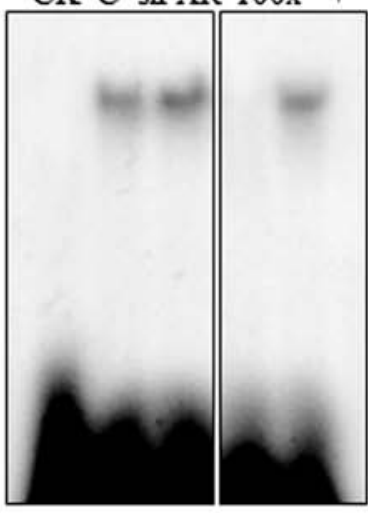

CK C siFXR 100x +

CK C siFXR 100x +

HT-29

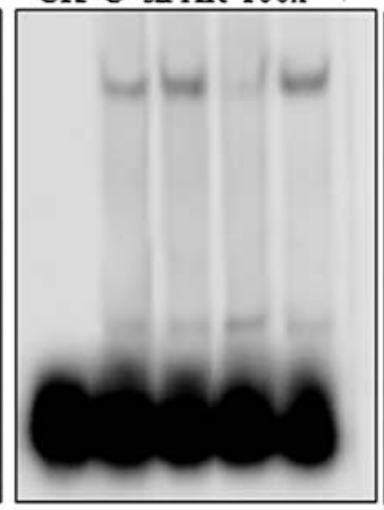

Caco-2
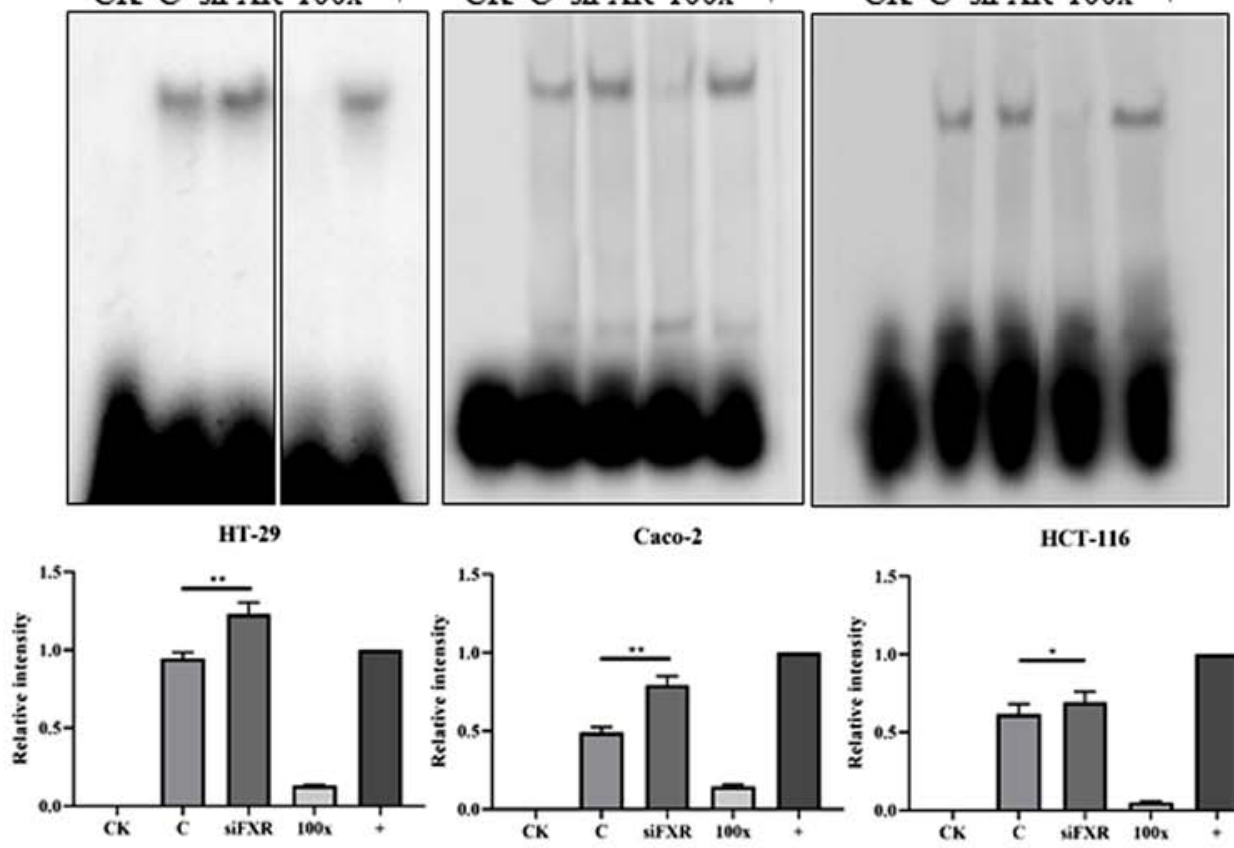

HCT-116

B

HT-29

Caco-2
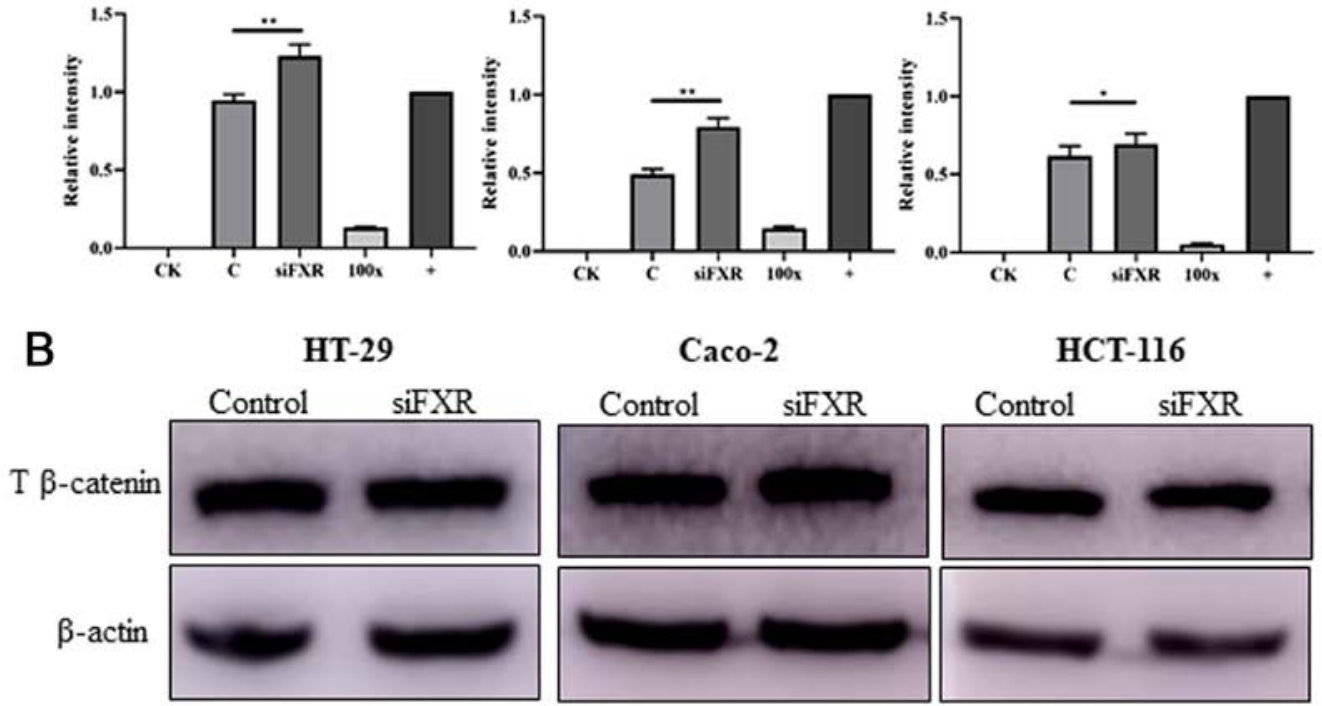

HCT-116
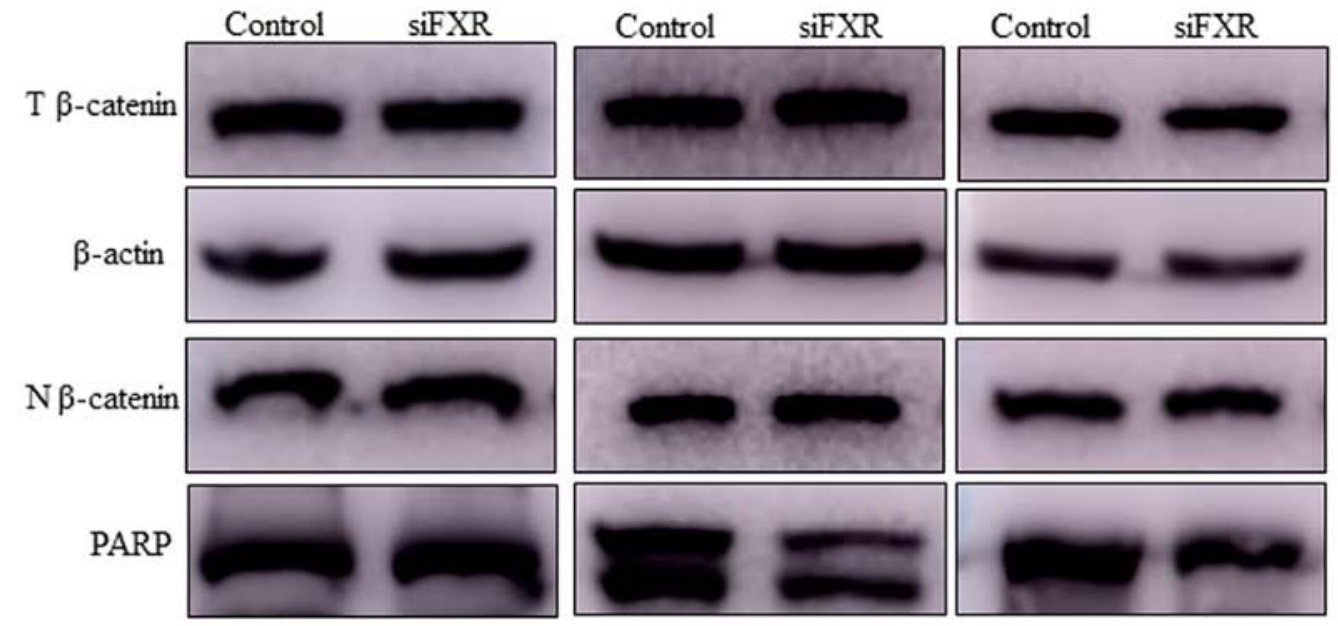

C
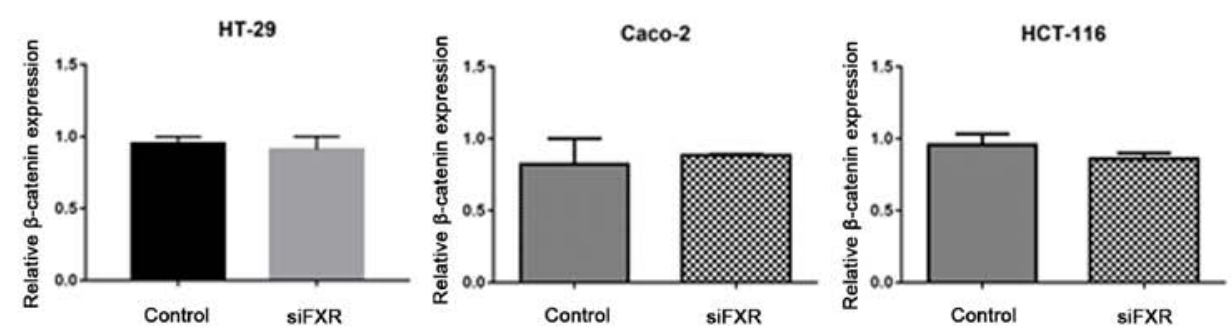

Figure 2. FXR activates the Wnt signaling pathway via increasing $\beta$-catenin/TCF4 complex levels. (A) Silencing FXR increases $\beta$-catenin/TCF4 complex formation as detected by an electrophoretic mobility shift assay. Images of HT-29 cells were from discontinuous parts of the same gel. Intensity analysis using ImageJ (version 1.52q; National Institute of Health) is also revealed to demonstrate the difference among groups. (B) $\mathrm{T}$ protein and $\mathrm{N}$ protein were extracted separately to determine the expression and distribution of $\beta$-catenin after FXR silencing. (C) $\beta$-catenin mRNA levels were not different after FXR silencing. PARP, Poly (ADP-ribose) polymerase, which is expressed in only the nucleus, was used as a loading control. The error bars represent the SEM of three independent samples. FXR, farnesoid X receptor; CK, background control; 100x, competitive control; +, positive control; C, control group pre-treated with negative control siRNA; $\mathrm{T}$, total; $\mathrm{N}$, nuclear; si, small interfering; NC, negative control. ${ }^{*} \mathrm{P}<0.05 ;{ }^{* *} \mathrm{P}<0.01$.

$\beta$-catenin-mediated transcriptional activity was subsequently tested using a cell-based TOPflash reporter assay. The TOPflash reporter, pRL-TK and siFXR or siNC were co-transfected into
HT-29, Caco-2 and HCT-116 cells. Silencing FXR significantly increased the $\beta$-catenin-mediated luciferase activity, driven by the TOPflash reporter, compared with negative control condi- 
A

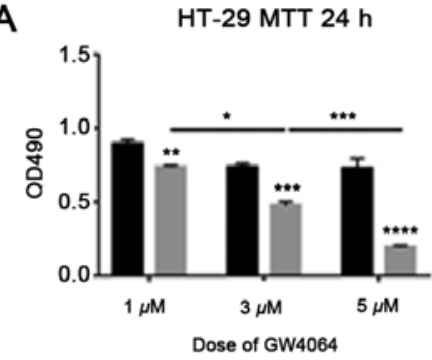

HT-29 MTT

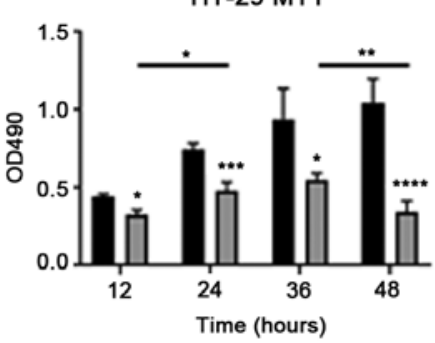

B
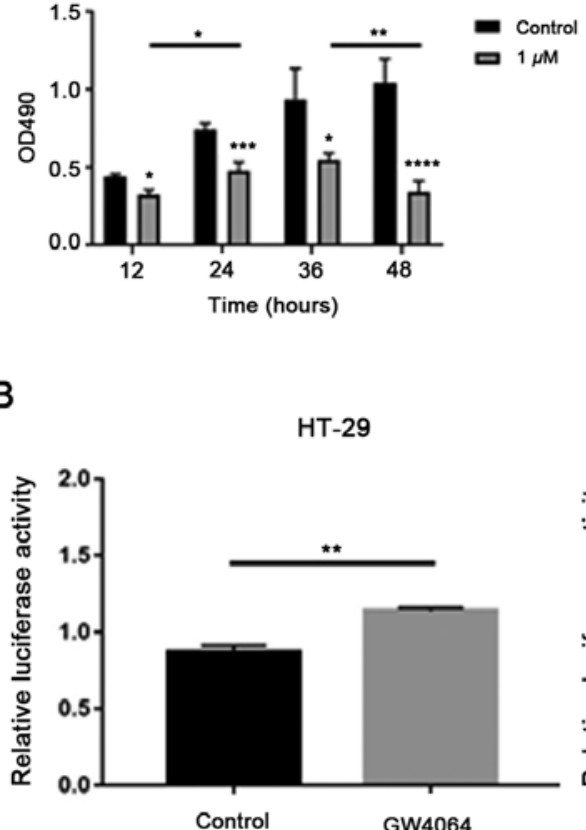

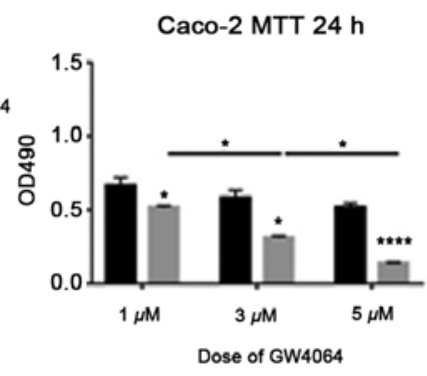

Caco-2 MTT

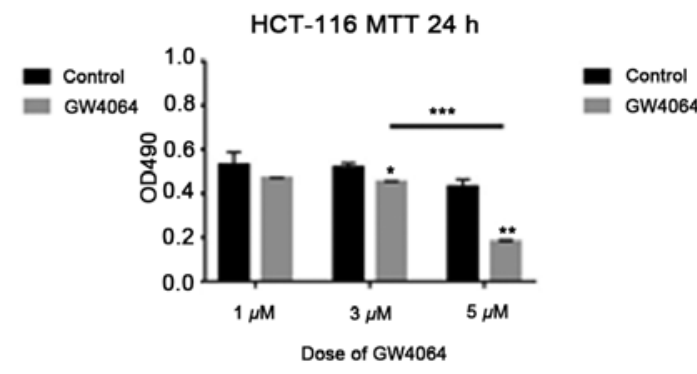

HCT-116 MTT
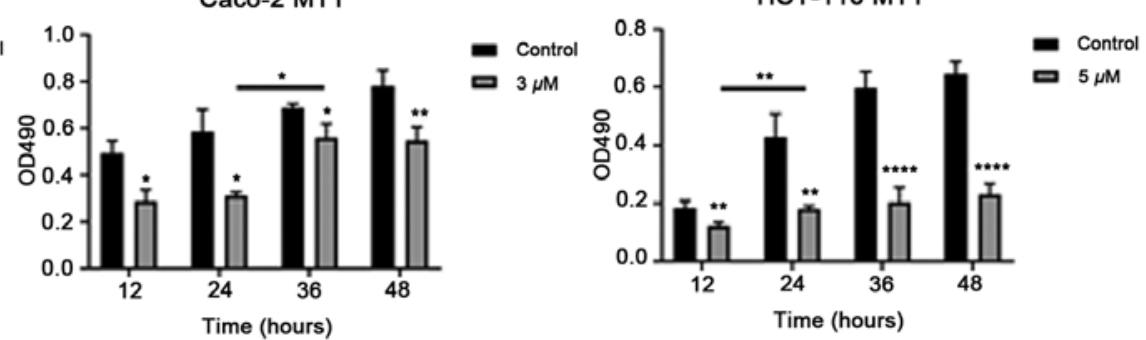

Caco-2

HCT-116
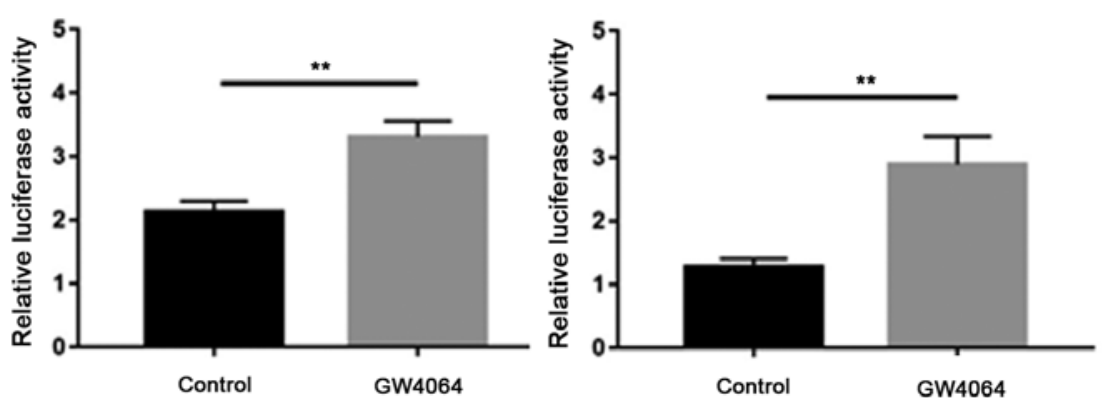

Figure 3. Effect of an FXR agonist on the Wnt signaling pathway. (A) FXR activation by GW4064 inhibits cell growth in a dose- and time-dependent manner as detected by MTT assay. (ANOVA, P<0.05) (B) Compared with the solvent control, GW4064 promotes $\beta$-catenin-mediated luciferase activity. The error bars represent the SEM of three independent samples. ${ }^{*} \mathrm{P}<0.05 ;{ }^{* *} \mathrm{P}<0.01 ;{ }^{* * *} \mathrm{P}<0.001 ;{ }^{* * * *} \mathrm{P}<0.0001$. FXR, farnesoid $\mathrm{X}$ receptor.

tions (Fig. 1C). These results suggested that FXR knockdown activates Wnt/ $\beta$-catenin transcriptional activity.

To explore the mechanism by which FXR interacts with the Wnt signaling pathway, the $\beta$-catenin/TCF4 complex levels were determined. Because the transcriptional activity of the Wnt signaling pathway is mediated by the $\beta$-catenin/TCF4 complex, EMSA was used to measure the active $\beta$-catenin/TCF4 complex levels. The silencing of FXR enhanced the formation of the $\beta$-catenin/TCF4 complex ( $\mathrm{P}<0.05$, Fig. $2 \mathrm{~A})$. However, the $\beta$-catenin protein distribution in the cytoplasm and nuclei was not different following FXR silencing (Fig. 2B). In addition, no difference was observed in $\beta$-catenin mRNA levels (Fig. 2C).

The current results indicated that silencing FXR activates the Wnt signaling pathway mediated by an increase in $\beta$-catenin/TCF4 complex levels, with no changes in the $\beta$-catenin mRNA and protein expression levels.

Effect of an FXR agonist on the Wnt signaling pathway. To confirm the appropriate dose and duration time of the FXR agonist GW4064, cell proliferation was measured using MTT assays. GW4064 significantly inhibited cell proliferation in a dose- and time-dependent manner compared with the solvent control, $(\mathrm{P}<0.05$, Fig. 3A). In HT-29 and Caco-2 cells with high FXR expression, $3 \mu \mathrm{M}$ GW4064 was used in the following experiments, and in HCT-116 cells with low FXR expression, $5 \mu \mathrm{M}$ GW4064 was used.

Subsequently, the effect of GW4064 on $\beta$-catenin-mediated transcriptional activity was tested. Cells were treated with GW4064 following transfection with TOPflash and pRL-TK. GW4064 promoted $\beta$-catenin-mediated luciferase activity compared with the solvent control (Fig. 3B).

FXR and $\beta$-catenin expression in a UC carcinogenesis mouse model. Mice treated with AOM/DSS exhibited significant body weight loss compared with control mice, following day 10 of administration $(\mathrm{P}=0.0001$, Fig. 4A). Colitis symptoms, such as loose and bloody stool, dull body hair, fatigue and decreased movement were accompanied, which were alleviated when the mice received ordinary drinking water. In weeks 9-10, two of the mice treated with AOM/DSS exhibited bloody stools again as well as anal prolapse, anal tumor fusion and ring growth at the end of the rectum (Fig. 4A). However, no apparent weight loss was observed among the groups at the end of the 12th week. Mice treated with AOM/DSS exhibited mucosal carcinoma or high-grade intraepithelial neoplasia, manifested as colonic gland structure disorder, large nuclei, deep staining and nucleoplasmic ratio imbalance (Fig. 4B). 

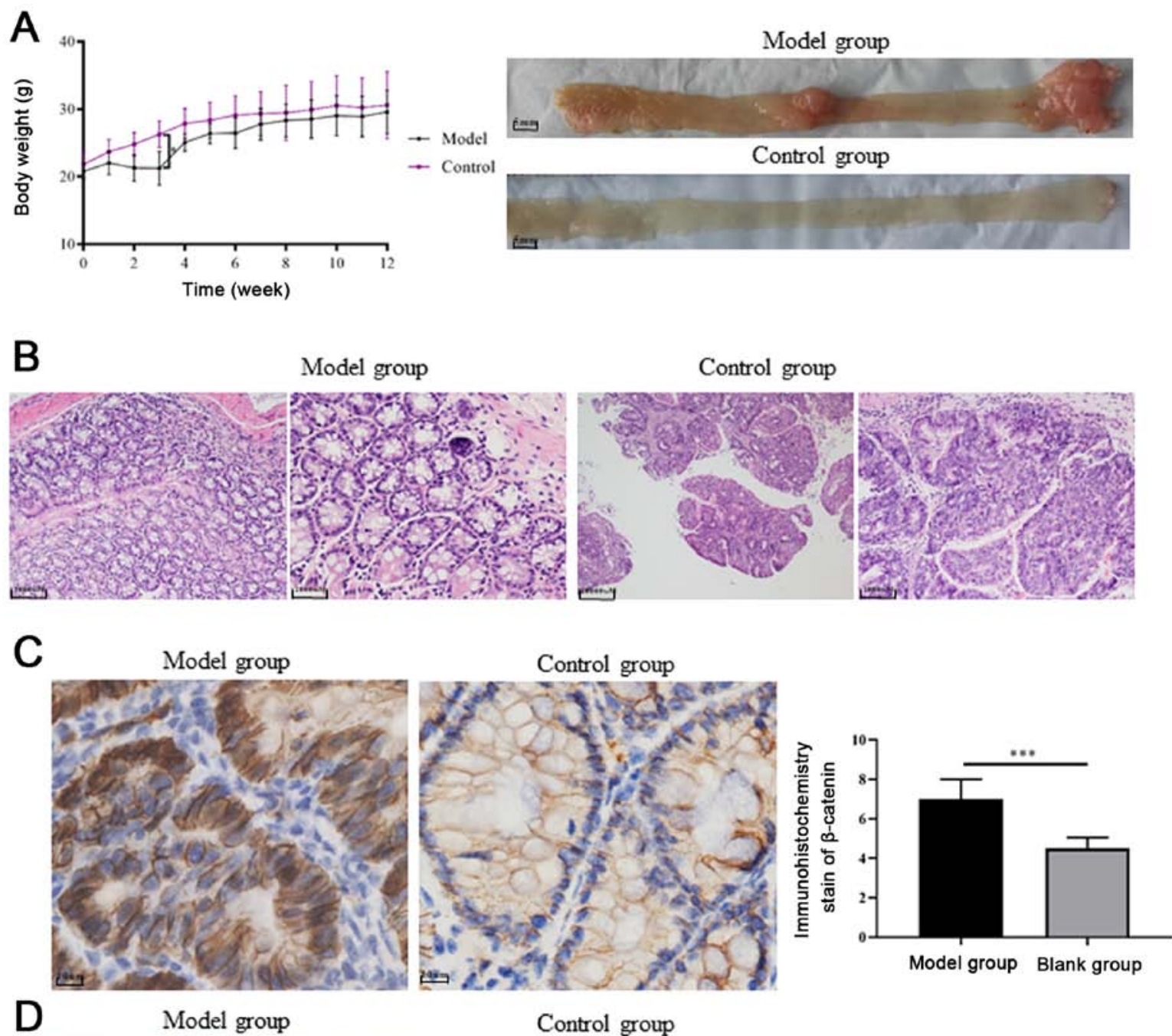

D
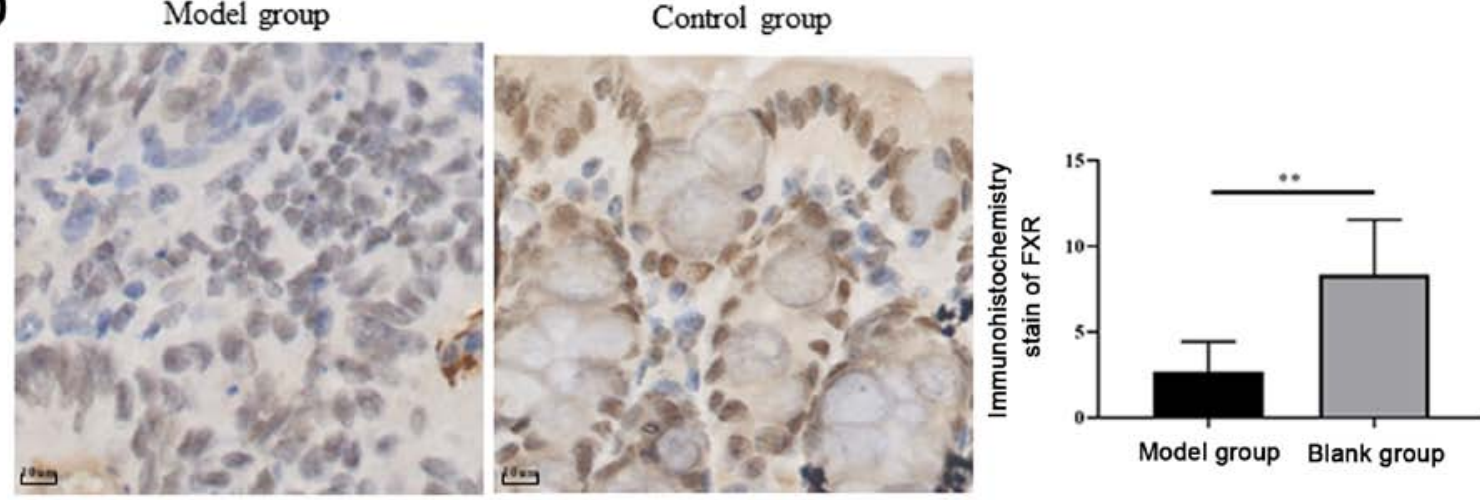

Figure 4. FXR and $\beta$-catenin expression in an ulcerative colitis carcinogenesis mouse model. (A) Representative images of colonic tumors in each group, model group exhibited anal prolapse, anal tumor fusion and ring growth at the end of the rectum. (B) Representative images of hematoxylin-eosin staining of colon tissue examined under a microscope (magnifications: Model group, $x 10$ and x20; control group, $x 4$ and x10). (C) Active $\beta$-catenin overexpression detected in tumor samples from the model group compared with the control group. (D) FXR expression was significantly lower in the model group compared with the control group. FXR, farnesoid $\mathrm{X}$ receptor. ${ }^{* *} \mathrm{P}<0.01$ and ${ }^{* * *} \mathrm{P}<0.001$.

In order to explore whether FXR and $\beta$-catenin activation influences chronic colitis-associated colorectal carcinoma development, the expression levels of FXR and $\beta$-catenin were evaluated in tissue samples from the UC carcinogenesis mouse model and control groups. The histopathological grades of FXR expression based on IHC staining scores were significantly lower in the model group compared with the control group ( $n=6 ; 2.667 \pm 0.7149$ vs. $8.333 \pm 1.308$, respectively; $\mathrm{P}<0.01$; Fig. 4D). By contrast, $\beta$-catenin upregulation was detected in the cell nucleus of tumor samples (Fig. 4C).

\section{Discussion}

Wnt/ $\beta$-catenin signaling is highly conserved from nematodes to humans, and is essential for various cellular processes, such as development, growth, survival, regeneration and self-renewal (23). Mutations in the Wnt/ $\beta$-catenin signaling 
pathway have been detected in $>90 \%$ of colon carcinogenesis cases (24). A close association was recently revealed between FXR and Wnt/ $\beta$-catenin signaling (25-27). FXR knockdown in a chronic colitis mouse model resulted in greater susceptibility to tumorigenesis and increased tumor progression (28). Correspondingly, increased expression of $\beta$-catenin and its downstream targets C-Myc and cyclin D1 was detected in FXR-deficient mice (28). Furthermore, FXR overexpression resulted in tumor growth suppression in both in FXR deficiency mice and colon cancer cell in vitro and in vivo (13).

In the present study, the crosstalk and mechanism between FXR and the Wnt signaling pathway was identified. FXR expression was significantly decreased and was inversely associated with $\beta$-catenin in both the mouse model and colon cancer cell lines. Consistent with the observations of the present study, FXR expression was also downregulated in mouse models and human CRC samples in previous studies $(7,8)$. In addition, a similar phenomenon was observed in cholangiocarcinoma, biliary tract carcinoma and tumor samples from patients with hepatocellular carcinoma (20), which may indicate the participation of BAs and FXR in the mechanism of these types of tumorigenesis. The results suggested that the interaction between FXR and $\beta$-catenin is a critical component in this process (20). Since previous studies have demonstrated a correlation between FXR deficiency and increased expression of $\beta$-catenin and its downstream targets C-Myc and cyclin D1, other stains that are associated with the development or growth of tumors have not been repeated.

In the present study, FXR knockdown significantly promoted $\beta$-catenin transcriptional activity. FXR appeared to regulate $\beta$-catenin activity via increasing $\beta$-catenin/TCF4 complex levels, without affecting $\beta$-catenin localization or total mRNA and protein levels. Activated $\beta$-catenin forms a complex with TCF4 and is recruited to the corresponding promoter region of Wnt target genes to elicit transcriptional activity (29). The results also suggest that FXR activation by GW4064 results in significantly inhibiting cell proliferation, and that GW4064 promoted $\beta$-catenin-mediated luciferase activity.

The present study demonstrated that the silencing of FXR by siRNA-FXR, and FXR activation by GW4084 treatment, both resulted in $\mathrm{Wnt} / \beta$-catenin signaling activation. This suggests that the interaction between FXR and the Wnt signaling pathway warrants further study. Consistent with the observations of the present study, previous studies have reported that FXR deficiency results in increased $\beta$-catenin transcriptional activity in chronic colitis mouse models $(13,28)$. However, few studies have focused on whether the effect of $\beta$-catenin occurs via FXR activation or upregulation. Only one study in the 293 cell line, which originates from renal epithelial cells, indicated that both GW4064-induced activation and FXR overexpression decreased $\beta$-catenin transcriptional activity (20). Since mutations in the Wnt signaling pathway have been detected in most colorectal cell lines, the effects of abnormal interaction sites may be relevant to the present study.

GW4064, a potent agonist of FXR, was used in the present study to explore the interaction between FXR and the Wnt signaling pathway. GW4064 is a synthetic isoxazole, which was used to decipher the cellular and physiological functions of FXR. Similar to previous studies $(20,21)$, the selected dose of GW4064 was $\sim 1-5 \mu \mathrm{M}$. In a study by Peng et al (21), the effect of GW4064 at $0.1,0.5,1,5$ and $10 \mu \mathrm{M}$ on cell proliferation was demonstrated in three colon cancer cell lines (H508, SNU-C4 and HT-29), and the results indicated that the effect was significant when the dose of GW4064 reaching $\sim 1 \mu \mathrm{M}$. However, in the physiological state, FXR is a member of the nuclear family and serves as a regulatory center of BAs. The complexity of the components of the BA pool and the crosstalk of nuclear family members may all contribute to its function $(6,7,14)$.

The crosstalk between $\mathrm{Wnt} / \beta$-catenin ligands and members of the nuclear receptor (NR) family has been considered a clinically and developmentally important research area of cancer biology (30). The NR family consists of retinoid X receptor (RXR), steroid receptor, peroxisome proliferator-activated receptor- $\gamma$ and vitamin D receptor (VDR). A high proportion of these proteins interact with the Wnt pathway via different mechanisms; for example, RXR binds $\beta$-catenin to degrade and decrease the levels of the $\beta$-catenin/TCF complex $(31,32)$. VDR activation increases VDR/ $\beta$-catenin complex formation, in addition to enhancing E-cadherin expression and sequestering TCF-bound $\beta$-catenin (33). The identification of functional interactions between Wnt signaling components and NRs is gaining increasing attention. A growing number of NRs appear to be activated by $\beta$-catenin, resulting in alterations in cell proliferation and tumorigenesis, whereas Wnt signaling appears to be compromised by the actions of NRs (30).

Exploration of the interaction of FXR with the Wnt/ $\beta$-catenin signaling pathway in the present study demonstrated that FXR knockdown promotes $\beta$-catenin/TCF4 complex formation and, subsequently, its binding ability to the corresponding promoter. The data indicate a novel mechanism through which FXR expression is mediated during tumor progression. Thus, FXR represents a novel modulator of the Wnt signaling pathway, and a potential molecular target of the Wnt signaling cascade that may be exploited to achieve antitumor effects.

\section{Acknowledgements}

Not applicable.

\section{Funding}

The present study was supported by grants from the National Natural Science Foundation of China (grant nos. 81370500 and 81770559).

\section{Availability of data and materials}

The datasets used and/or analyzed during the current study are available from the corresponding author on reasonable request.

\section{Authors' contributions}

JL designed the study. JM and XC performed the cell experiments, and CW and WL performed the animal experiments. WL helped interpret the data. JM and XC analyzed the data. JM wrote the manuscript. CW revised the manuscript for important intellectual content. All authors read and approved the final manuscript. 


\section{Ethics approval and consent for participate}

All animal experiments were approved by and conducted in accordance with the recommendations of the Animal Care Ethics and Use Committee of Peking Union Medical College (approval no. XHDW-2015-0032).

\section{Patient consent for publication}

Not applicable.

\section{Competing interests}

The authors declare that they have no competing interests.

\section{References}

1. Reddy BS: Diet and excretion of bile acids. Cancer Res 41 3766-3768, 1981

2. Lagergren J, Ye W and Ekbom A: Intestinal cancer after cholecystectomy: Is bile involved in carcinogenesis? Gastroenterology 121: 542-547, 2001.

3. Peterlik M: Role of bile acid secretion in human colorectal cancer. Wien Med Wochenschr 158: 539-541, 2008.

4. Pearson JR, Gill CI and Rowland IR: Diet, fecal water, and colon cancer-development of a biomarker. Nutr Rev 67: 509-526, 2009.

5. Kundu S, Kumar S and Bajaj A: Cross-talk between bile acids and gastrointestinal tract for progression and development of cancer and its therapeutic implications. IUBMB Life 67: 514-523, 2015.

6. Degirolamo C, Modica S, Palasciano G and Moschetta A: Bile acids and colon cancer: Solving the puzzle with nuclear receptors. Trends Mol Med 17: 564-572, 2011.

7. Modica S, Gofflot F, Murzilli S, D'Orazio A, Salvatore L, Pellegrini F, Nicolucci A, Tognoni G, Copetti M, Valanzano R, et al: The intestinal nuclear receptor signature with epithelial localization patterns and expression modulation in tumors. Gastroenterology 138: 636-648, 648.e1-12, 2010.

8. Bailey AM, Zhan L, Maru D, Shureiqi I, Pickering CR, Kiriakova G, Izzo J, He N, Wei C, Baladandayuthapani V, et al: FXR silencing in human colon cancer by DNA methylation and KRAS signaling. Am J Physiol Gastrointest Liver Physiol 306 : G48-G58, 2014

9. Lax S, Schauer G, Prein K, Kapitan M, Silbert D, Berghold A, Berger A and Trauner M: Expression of the nuclear bile acid receptor/farnesoid $\mathrm{X}$ receptor is reduced in human colon carcinoma compared to nonneoplastic mucosa independent from site and may be associated with adverse prognosis. Int J Cancer 130: 2232-2239, 2012.

10. Frankenberg T, Rao A, Chen F, Haywood J, Shneider BL and Dawson PA: Regulation of the mouse organic solute transporter alpha-beta, Ostalpha-Ostbeta, by bile acids. Am J Physiol Gastrointest Liver Physiol 290: G912-G922, 2006.

11. De Gottardi A, Touri F, Maurer CA, Perez A, Maurhofer O, Ventre G, Bentzen CL, Niesor EJ and Dufour JF: The bile acid nuclear receptor FXR and the bile acid binding protein IBABP are differently expressed in colon cancer. Dig Dis Sci 49: 982-989, 2004.

12. Gadaleta RM, van Erpecum KJ, Oldenburg B, Willemsen EC, Renooij W, Murzilli S, Klomp LW, Siersema PD, Schipper ME, Danese S, et al: Farnesoid X receptor activation inhibits inflammation and preserves the intestinal barrier in inflammatory bowel disease. Gut 60: 463-472, 2011.

13. Modica S, Murzilli S, Salvatore L, Schmidt DR and Moschetta A: Nuclear bile acid receptor FXR protects against intestinal tumorigenesis. Cancer Res 68: 9589-9594, 2008

14. Gadaleta RM, Garcia-Irigoyen O and Moschetta A: Bile acids and colon cancer: Is FXR the solution of the conundrum? Mol Aspects Med 56: 66-74, 2017.

15. Korinek V, Barker N, Morin PJ, van Wichen D, de Weger R, Kinzler KW, Vogelstein B and Clevers H: Constitutive transcriptional activation by a beta-catenin-Tcf complex in APC-/-colon carcinoma. Science 275: 1784-1787, 1997.
16. Mizushima T, Nakagawa H, Kamberov YG, Wilder EL, Klein PS and Rustgi AK: Wnt-1 but not epidermal growth factor induces beta-catenin/T-cell factor-dependent transcription in esophageal cancer cells. Cancer Res 62: 277-282, 2002

17. Ni D, Liu J, Hu Y, Liu Y, Gu Y, Zhou Q and Xie Y: A1CF-Axin2 signal axis regulates apoptosis and migration in Wilms tumor-derived cells through Wnt $/ \beta$-catenin pathway. In Vitro Cell Dev Biol Anim 55: 252-259, 2019.

18. Xin Y, He L, Luan Z, Lv H, Yang H, Zhou Y, Zhao X, Zhou W, Yu S, Tan B, et al: E-cadherin mediates the preventive effect of Vitamin D3 in Colitis-associated Carcinogenesis. Inflamm Bowel Dis 23: 1535-1543, 2017.

19. Livak KJ and Schmittgen TD: Analysis of relative gene expression data using real-time quantitative PCR and the 2(-Delta Delta C(T)) method. Methods 25: 402-408, 2001

20. Liu X, Zhang X, Ji L, Gu J, Zhou M and Chen S: Farnesoid X receptor associates with $\beta$-catenin and inhibits its activity in hepatocellular carcinoma. Oncotarget 6: 4226-4238, 2015.

21. Peng Z, Raufman JP and Xie G: Src-mediated cross-talk between farnesoid $\mathrm{X}$ and epidermal growth factor receptors inhibits human intestinal cell proliferation and tumorigenesis. PLoS One 7: e48461, 2012.

22. Tanaka T, Kohno H, Suzuki R, Yamada Y, Sugie S and Mori H: A novel inflammation-related mouse colon carcinogenesis model induced by azoxymethane and dextran sodium sulfate. Cancer Sci 94: 965-973, 2003.

23. Cavard C, Colnot S, Audard V, Benhamouche S, Finzi L, Torre C, Grimber G, Godard C, Terris B and Perret C: Wnt/beta-catenin pathway in hepatocellular carcinoma pathogenesis and liver physiology. Future Oncol 4: 647-660, 2008

24. Cancer Genome Atlas Network: Comprehensive molecular characterization of human colon and rectal cancer. Nature 487 330-337, 2012.

25. Fu T, Coulter S, Yoshihara E, Oh TG, Fang S, Cayabyab F, Zhu Q, Zhang T, Leblanc M, Liu S, et al: FXR regulates intestinal cancer stem cell proliferation. Cell 176: 1098-1112.e18, 2019.

26. Thompson MD, Moghe A, Cornuet P, Marino R, Tian J, Wang P, Ma X, Abrams M, Locker J, Monga SP and Nejak-Bowen K: $\beta$-Catenin regulation of farnesoid $X$ receptor signaling and bile acid metabolism during murine cholestasis. Hepatology 67: 955-971, 2018.

27. Zhang R, Nakao T, Luo J, Xue Y, Cornuet P, Oertel M, Kosar K, Singh $\mathrm{S}$ and Nejak-Bowen K: Activation of WNT/Beta-catenin signaling and regulation of the Farnesoid $X$ Receptor/Beta-catenin complex after murine bile duct ligation. Hepatol Communications 3: 1642-1655, 2019.

28. Maran RR, Thomas A, Roth M, Sheng Z, Esterly N, Pinson D, Gao X, Zhang Y, Ganapathy V, Gonzalez FJ and Guo GL: Farnesoid $X$ receptor deficiency in mice leads to increased intestinal epithelial cell proliferation and tumor development. J Pharmacol Exp Ther 328: 469-477, 2009.

29. Sawa M, Masuda M and Yamada T: Targeting the Wnt signaling pathway in colorectal cancer. Expert Opin Ther Targets 20: 419-429, 2016.

30. Mulholland DJ, Dedhar S, Coetzee GA and Nelson CC: Interaction of nuclear receptors with the Wnt $/ \beta$-catenin/Tcf signaling axis: Wnt you like to know? Endocr Rev 26: 898-915, 2006.

31. Xiao JH, Ghosn C, Hinchman C, Forbes C, Wang J, Snider N, Cordrey A, Zhao Y and Chandraratna RA: Adenomatous polyposis coli (APC)-independent regulation of beta-catenin degradation via a retinoid X receptor-mediated pathway. J Biol Chem 278: 29954-29962, 2003.

32. Han A, Tong C, Hu D, Bi X and Yang W: A direct protein-protein interaction is involved in the suppression of beta-catenin transcription by retinoid $\mathrm{X}$ receptor alpha in colorectal cancer cells. Cancer Biol Ther 7: 454-459, 2008.

33. Palmer HG, González-Sancho JM, Espada J, Berciano MT, Puig I, Baulida J, Quintanilla M, Cano A, de Herreros AG, Lafarga M and Muñoz A: Vitamin D(3) promotes the differentiation of colon carcinoma cells by the induction of E-cadherin and the inhibition of beta-catenin signaling. J Cell Biol 154: 369-387, 2001.

This work is licensed under a Creative Commons Attribution-NonCommercial-NoDerivatives 4.0 International (CC BY-NC-ND 4.0) License. 